\title{
Best Practices for Clinical Evaluation of Sacroiliac Joint Pain: An Evidence-to-Practice Review
}

Nicolette A. Harris, DAT, LAT, ATC, CSCS*; Adriana Peña, MS, LAT, ATC†; and Sofia Núñez Rivera, MS, LAT, ATC

*Florida International University, Miami; † Sports Leadership and Management Academy, Miami, FL

\begin{abstract}
Sacroiliac joint (SIJ) pain serves as an under-recognized source of chronic low back pain. Improvement in the accuracy of a clinical SIJ pain diagnosis lends a higher likelihood of appropriate treatment measures, better patient outcomes and decreased out-of-pocket costs. Therefore, the overall purpose of this evidence to practice review was to highlight the main points of a systematic review on the clinical diagnosis of SIJ pain. Searches of five electronic databases revealed 758 studies, nonetheless only six studies met final inclusion criteria. Studies included were assessed by the authors for methodological quality using the Quality Assessment of Diagnostic Accuracy Studies (QUADAS) tool. Evidence suggests pain provocation tests including distraction, thigh thrust, compression, sacral thrust, and Gaenslen's are minimally useful individually at diagnosing sacroiliac joint pain. The thigh thrust test was the most sensitive and the distraction test was most specific. Furthermore, the compression test carried the strongest positive likelihood ratio. The highest likelihood ratio was reported when three or more of the following pain provocation tests were positive: distraction, compression, thigh thrust, sacral thrust, and Gaenslen's test for both the right and left sides. A comparable likelihood ratio was found when any two of the remaining four tests were positive after excluding the left and right-side applications of the Gaenslen's test. Prior to the performance of pain provocation tests, research suggests using McKenzie Mechanical Diagnosis and Therapy to exclude pain of disc origin. The use of safe, efficient, and clinically effective diagnostic evaluation techniques is essential to the provision of high-quality patient care.
\end{abstract}

Key Phrases

Diagnostic testing and physical examination: spine, thorax, and abdomen; low back pain; sacroiliac joint

\section{Correspondence}

Dr. Nicolette A Harris, Florida International University,

11200 SW 8th St, WRC 145, Miami, FL 33199.

E-mail: nstallwo@fiu.edu

Twitter: @YoungProf_AT

\section{Full Citation}

Harris NA, Peña A, Núñez Rivera S. Best practices for clinical evaluation of sacroiliac joint pain: An evidence to practice review. Clin Pract Athl Train. 2021;4(1): 17-23. https://doi.org/10.31622/2021/0004.1.3.

Submitted: August 17, 2020 Accepted: October 1, 2020.

Copyright (C) by Indiana State University All rights reserved. ISSN Online 2577-8188

\section{ORIGINAL REFERENCE}

Sivayogam A, Baneriee A. Diagnostic performance of clinical tests for sacroiliac joint pain. Phys Ther Rev. 201 1;16(6):462-467.

\section{SUMMARY}

\section{CLINICAL PROBLEM AND QUESTION}

\begin{abstract}
$A_{p}$ pproximately $10-30 \%$ of all low back pain is originated from the sacroiliac joint (SIJ). ${ }^{1}$ The SIJ is a large, auricular-shaped, and arthrodial synovial joint formed by the connection of the sacrum to the right and left iliac bones. ${ }^{2}$ The primary function of the SIJ is to absorb shock and transfer forces between the upper and lower extremities. ${ }^{3}$ More extensive dorsally, the ligaments of the SIJ function to limit motion in all planes. ${ }^{3}$ While the SIJ lacks significant range of motion, patients may possess hypomobility or hypermobility of the SIJ articulation. ${ }^{2}$ The most common mechanism for acute SIJ pain results from a combination of axial compression and rapid rotation, such as with twisting while carrying a heavy object or falling. ${ }^{1}$ However, most athletes will experience a slow and progressive onset of symptoms resulting from repetitive activity performed over time. ${ }^{1}$ Common pathologies arising from the SIJ include: sprains, strains and dysfunction of the joint secondary to insufficient or excessive mobility. ${ }^{1}$ Factors that increase the risk of these conditions include leg length discrepancy, antalgic gait, scoliosis, and prolonged vigorous exercise. ${ }^{1}$ This multitude of triggers, makes low back pain of SIJ origin extremely challenging for the health care provider to clinically diagnose.
\end{abstract}


Regrettably, no single history presentation, clinical examination finding, or diagnostic imaging technique can definitely establish a diagnosis of sacroiliac joint pain. ${ }^{4}$ However, previous research has studied these measures in an effort to improve accuracy in the diagnosis of SIJ pain. ${ }^{5}$ While many publications have referenced controlled local anesthetic blocks as the best available tool to determine the source of SIJ pain, these measures are invasive and expensive, making them clinically unpractical for routine use in the ambulatory care setting. ${ }^{5}$ There are a variety of "special" tests used by clinicians in the evaluation and diagnosis of SIJ pain. These include motion palpation and pain provocation tests. ${ }^{6}$ Numerous studies have proposed palpation as a method to assess movement or asymmetry at the SIJ.7 However, generally their inter-examiner reliability has shown poor.7 This leaves clinicians to rely on pain provocation tests, which stress the structures of the SIJ and provoke reproduction of symptoms, as measures of non-invasive clinical evaluation of SIJ pain.7 Common pain provocation tests used for clinical evaluation include distraction, compression, sacral thrust, thigh thrust, and Gaenslen's tests in addition to a host of others.7 The diagnostic accuracy of pain provocation tests has been called into question for its inability to discriminate pain of sacroiliac origin as compared to the reference standard. 7 Consequently, clinicians must be aware of both the sensitivity, or ability to distinguish subjects with the disease, as well as the specificity, or the ability to identify patients without the disease associated with the tests used for effective diagnosis of SIJ pain. ${ }^{8}$

Therefore, the overall purpose of the guiding paper was to systematically review and synthesize evidence associated with the clinical diagnosis of SIJ pain. More precisely, this Evidence to Practice Review aims to remedy the following research question: In patients with low back pain, which clinical evaluation tests are most accurate for diagnosing pain of sacroiliac origin?

Copyright (C) by Indiana State University All rights reserved. ISSN Online 2577-8188

\section{SUMMARY OF LITERATURE}

Authors of the systematic review performed a methodical search of MEDLINE, Scopus, AMED, CINAHL, and EMBASE databases to determine the diagnostic performance of clinical tests for SIJ pain. Searches were filtered to include only articles published in English between 1990 and 2011. Studies specifically addressing SIJ dysfunction rather than SIJ pain were excluded. Single articles were included in the systematic review according to the following criteria: (1) patients were at least 18 years old, (2) had nonspecific and non-pregnancy related low back pain and/or buttock pain with or without radiation into the lower extremity, (3) used clinical tests with clear definitions of positive and negative test results, and (4) provided sensitivity and specificity data. Initially 758 studies were identified, however 752 were excluded for failing to meet selection criteria. Of the six studies included, two studies evaluated the validity of each individual test; three studies evaluated the validity of several composites of tests; and one study evaluated the validity of both individual and composites of tests. All six of the studies included used a contrast-enhanced intra-articular anesthetic block as the reference standard. The number of patients enclosed in the studies ranged from 34 to 140 with a mean age between 42 and 51 years old.

\section{SUMMARY OF OUTCOMES}

Studies included in the guiding systematic review were assessed for methodological quality autonomously by both authors using the Quality Assessment of Diagnostic Accuracy Studies (QUADAS) tool. The QUADAS tool consists of 14 items which can be answered with a yes, no, or unclear. Nine of the 12 items relate to bias, while three of the 12 items related to the quality of the reporting and two of the 12 items conveyed variability. Intra-articular administration of an anesthetic block into the SIJ bilaterally was 
agreed upon by the authors as the best available reference standard for fulfilling QUADAS item number 3 and correctly classifying SIJ pain. Furthermore, to achieve item number 4 of the QUADAS and to rule out spontaneous recovery or progression to more severe pain, the acceptable gap was agreed on by both authors as no more than seven days between performance of clinical and reference tests. Items $1,5,10,11$, and 12 were scored using a 3 for yes; items 3 and 6 were marked 2 for yes; and all other items were counted 1 for yes. ${ }^{6}$ A quality score of 17 was assigned to five of 6 items, while a quality score of 18 was allocated to the sixth study. ${ }^{6}$

\section{FINDINGS AND CLINCIAL IMPLICATIONS}

The systematic review guiding this paper aimed to assess the diagnostic performance of clinical tests commonly used to evaluate SIJ pain. For each clinical test, sensitivity and specificity were documented. In addition, positive predictive values (PPV) were included to describe how often a positive finding was correct, while negative predictive values (NPV) were provided to consider the accuracy of a negative test result. Furthermore, positive and negative likelihood ratios were extracted when available. Positive likelihood ratios $(L R+)$ were used to provide confidence in the fact that the pathology was present when a test was positive. Likewise, negative likelihood ratios (LR-) express the probability that the condition was present despite a negative result on the diagnostic test. Evidence revealed that when used in isolation, most clinical tests had poor diagnostic performance. ${ }^{9}$ Clinical tests including the Gillet, pain over SIJ or groin or buttock, sitting position, posterior superior iliac spine pointing, sacral spring, and sacral sulcus revealed poor clinical utility as result of low specificity, low sensitivity, and positive likelihood ratio. ${ }^{9}$ However, the high sensitivity and specificity values of FABER, thigh thrust, and resisted abduction may make these tests better indicators of SIJ pathology. ${ }^{10}$ Furthermore, evidence

Copyright $\left({ }^{\circ}\right.$ by Indiana State University All rights reserved. ISSN Online 2577-8188 suggests pain provocation tests including distraction, compression, thigh thrust, sacral thrust, and Gaenslen's are not effective predictors of a positive intra-articular SIJ anesthetic block when used alone without any other tests. "1 However the thigh thrust test was most sensitive, while distraction test was most specific and compression test carried the strongest positive likelihood ratio (Table 1).6,11

Clinicians should choose a composite of tests which may strengthen the likelihood of an accurate clinical diagnosis. Evidence suggests an optimal ratio of pain provocation tests composites which can provide high specificity paired with low sensitivity while still maintaining a high likelihood ratio.11, 12 The highest likelihood ratio was reported when three or more of the following pain provocation tests were positive: distraction, compression, thigh thrust, sacral thrust, and Gaenslen's test for both the right and left sides. ${ }^{11}$ A comparable likelihood ratio was found when any two of the remaining four tests were positive after eliminating the left and right-side applications of the Gaenslen's test (Figure 1).11 The inclusion of positive and negative likelihood ratios in the individual studies helped to formulate the conclusion that when in the presence of pain below the lumbosacral region or groin, the three prime tests for diagnosis for SIJ pain are distraction, thigh thrust and compression. ${ }^{6}$ Only after all six SIJ pain provocation tests are negative, can SIJ pain be ruled out. " Using clinical examination techniques with high diagnostic accuracy can eliminate or significantly reduce the need to refer patients for diagnostic imaging. Safe, effective, and efficient diagnostic techniques can help improve the quality of care. Furthermore, the use of clinical evaluation techniques can decrease health care costs, making care more equitable for everyone.

To further reduce false positives, research suggests using the McKenzie evaluation to exclude pain of disc origin prior to the performance of

Clinical Practice in Athletic Training Volume 4 - Issue 1 - February 2021 
Table 1. Summary of Diagnostic Accuracy for Individual Clinical Tests of Sacroiliac Joint Pain

\begin{tabular}{|c|c|c|c|c|}
\hline Intervention & Study & Sensitivity $(95 \% \mathrm{Cl})$ & Specificity $(95 \% \mathrm{Cl})$ & $+\mathrm{LR}^{\dagger}$ \\
\hline \multirow[t]{3}{*}{ Thigh thrust } & Dreyfuss et al. ${ }^{8}$ & 0.36 & 0.50 & \\
\hline & $\begin{array}{l}\text { Broadhurst and } \\
\text { Bond } 9\end{array}$ & 0.80 & 1.00 & \\
\hline & Laslett et al. ${ }^{10}$ & $0.88(0.64-0.97)$ & $0.69(0.82)$ & 2.20 \\
\hline \multirow[t]{3}{*}{ Gaenslen's } & Dreyfuss et al. ${ }^{8}$ & 0.71 & 0.26 & \\
\hline & Laslett et al. ${ }^{10}$ & $0.53(0.30-0.75) R$ & $0.71(0.53-0.84) R$ & 1.84 \\
\hline & & $0.50(0.27-0.73) \mathrm{L}$ & $0.77(0.60-0.89) \mathrm{L}$ & 2.21 \\
\hline \multirow[t]{2}{*}{ Sacral thrust } & Dreyfuss et al. ${ }^{8}$ & 0.53 & 0.29 & \\
\hline & Laslett et al. 10 & $0.63(0.39-0.82)$ & $0.75(0.58-0.87)$ & 2.50 \\
\hline Distraction test & Laslett et al. ${ }^{10}$ & $0.60(0.36-0.80)$ & $0.81(0.65-0.91)$ & 3.20 \\
\hline Compression & Laslett et al. ${ }^{10}$ & $0.69(0.44-0.86)$ & $0.69(0.51)$ & 2.20 \\
\hline
\end{tabular}

Note: * ${ }^{\mathrm{Cl}}$ : confidence interval.

$\dagger+L R$ : positive likelihood ratio.

pain provocation tests. ${ }^{13}$ McKenzie Mechanical Diagnosis and Therapy (MDT) is a well-studied technique which utilizes repeated movements to assess musculoskeletal disorders of the spine and extremities. ${ }^{14}$ By performing repeated movements during the examination, patients may develop a direction of preference (e.g., truck flexion, extension, or lateral bending) that is correlated to a movement which centralizes the pain from the extremities to the spinal midline. ${ }^{14,7}$ Centralization has been reported as highly specific to discogenic pain, yet is not observed in patients with confirmed pain of sacroiliac origin. ${ }^{7}$ Therefore, we strongly recommend the prerequisite use of MDT by clinicians to rule out discogenic pain prior to evaluation of the SIJ using pain provocation tests. Moreover, current research suggests MDT can also be used in adjunct to pain provocation tests to as a method of alleviating pain and further strengthening SIJ pain diagnoses. ${ }^{14}$ Patients may be further classified with SIJ pain when repeated anterior and posterior innominate rotation movements alleviate pain and symptoms from the region of the posterior superior iliac spine (PSIS). ${ }^{14}$

Copyright (C by Indiana State University All rights reserved. ISSN Online 2577-8188

\section{CLINICAL BOTTOM LINE}

Diagnostic tests are a critical component of health care. Information on the accuracy of diagnostic tests can help in clinical decision-making and assist in the provision of safe, timely, effective, efficient, and equitable care. Based on the findings of this review, we suggest a framework for improved diagnosis in the evaluation of SIJ pain. Evaluation of pain below the lumbosacral or groin region should begin with a thorough patient history. Moving forward, a McKenzie MDT evaluation assessing for centralization should be performed to rule out pain of discogenic origin. This should be proceeded by the performance of 6 pain provocation tests: beginning with distraction, thigh thrust, and compression tests and continuing with the sacral thrust and Gaenslen's test for both the right and left sides as needed. The receipt of 3 or more positive tests provide the optimal balance between high specificity, low sensitivity, and a high likelihood ratio. We believe use of this criterion is best practice for establishing a highly accurate SIJ pain diagnosis. Alleviation of the patient's symptoms through repeated anterior and posterior innominate rotation may further validate diagnosis, but more research is needed to support 
this recommendation. It is our view that improved diagnostic accuracy will lead to a higher likelihood of appropriate treatment measures, resulting in decreased out-of-pocket costs, improved patient care, and enhanced patient outcomes.

\section{REFERENCES}

1. Peebles $R$, Jonas CE. Sacroiliac joint dysfunction in the athlete: Diagnosis and Management. Curr Sports Med Rep. 2017; 16(5):336-342.

https://doi.org/10.1249/JSR.0000000000 $\underline{000410 .}$

2. Cohen SP. Sacroiliac joint pain: a comprehensive review of anatomy, diagnosis, and treatment. Anesth Analg. 2005; $101(5): 1440-53$.

3. Vleeming A, Schuenke MD, Masi AT, Carreiro JE, Danneels L, Willard FH. The sacroiliac joint: an overview of its anatomy, function and potential clinical implications. J Anat. $2012 ; 221(6): 537-67$.

https://doi.org/10.1213/01.ANE.0000180 831.60169. EA.

4. Chauhan G, Hehar P, Loomba V, Upadhyay A. A Randomized Controlled Trial of Fluoroscopically Guided Sacroiliac Joint Injections: A Comparison of the Posteroanterior and Classical Oblique Techniques. Neurospine. 2019;16(2):317324.

5. Simopoulos TT, Manchikanti L, Gupta S, et al. Systematic Review of the Diagnostic accuracy and therapeutic effectiveness of sacroiliac joint interventions. Pain Physician. 2015; 18(5): E713-56. https://pubmed.ncbi.nlm.nih.gov/26431129 L.

6. Sivayogam A, Banerjee A. Diagnostic performance of clinical tests for sacroiliac

Copyright $(\subseteq$ by Indiana State University All rights reserved. ISSN Online 2577-8188 joint pain. Phys Ther Rev. 2011;16(6):462467.

https://doi.org/10.1179/1743288X11Y.00 00000036 .

7. Laslett M. Evidence-based diagnosis and treatment of the painful sacroiliac joint. J Man Manip Ther. 2008;16(3):142-52. https://doi.org/10.1179/imt.2008.16.3.14 $\underline{2}$.

8. Šimundić AM. Measures of diagnostic accuracy: basic definitions. EJIFCC. 2009; 19(4):203-11.

https://www.ncbi.nlm.nih.gov/pmc/articles/P MC4975285/.

9. Dreyfuss P, Michaelsen M, Pauza K, McLarty J, Bogduk N. The value of medical history and physical examination in diagnosing sacroiliac joint pain. Spine 1996; 21:2594-602. https://doi.org/10.1097/00007632$199611150-00009$.

10. Broadhurst NA, Bond MJ. Pain provocation tests for the assessment of sacroiliac joint dysfunction. J Spinal Disord 1998; 1 1:341-5. https://pubmed.ncbi.nlm.nih.gov/9726305/.

11. Laslett $M$, Aprill C, McDonald B, Young S. Diagnosis of sacroiliac joint pain: validity of individual provocation tests and composites of tests. Man Ther. 2005; 10:207-18. https://doi.org/10.1016/i.math.2005.01.00 3.

12. van der Wurff $P$, Buijs EJ, Groen GJ. A multitest regimen of pain provocation tests as an aid to reduce unnecessary minimally invasive sacroiliac joint procedures. Arch Phys Med Rehabil 2006; 87:10-4. https://doi.org/10.1016/i.apmr.2005.09.0 $\underline{23}$.

13. Laslett M, Young SB, Aprill CN, Mcdonald B. Diagnosing painful sacroiliac joints: A validity study of a McKenzie evaluation and sacroiliac

Clinical Practice in Athletic Training Volume 4 - Issue 1 - February 2021 
provocation tests. Aust J Physiother. 2003;49(2):89-97.

https://doi.org/10.1016/s0004-

9514(14)60125-2.

14. Srivastava S, Kumar DK, Mittal H, Dixit S. Short-term effect of "Mechanical Diagnosis and Therapy" in the management of sacroiliac joint pain. I Clin Diagn Res. 2018;12(8): 1-4.

15. Petersen T, Laslett $M$, Juhl C. Clinical classification in low back pain: best-evidence diagnostic rules based on systematic reviews. BMC Musculoskelet Disord. 2017;18(1):188.

16. Stanford G, Burnham RS. Is it useful to repeat sacroiliac joint provocative tests post-block? Pain Med 2010; 11:1774-6. https://doi.org/10.1111/i.15264637.2010.00968.x. 
Figure 1. Diagnostic Algorithm for Sacroiliac Joint Pain

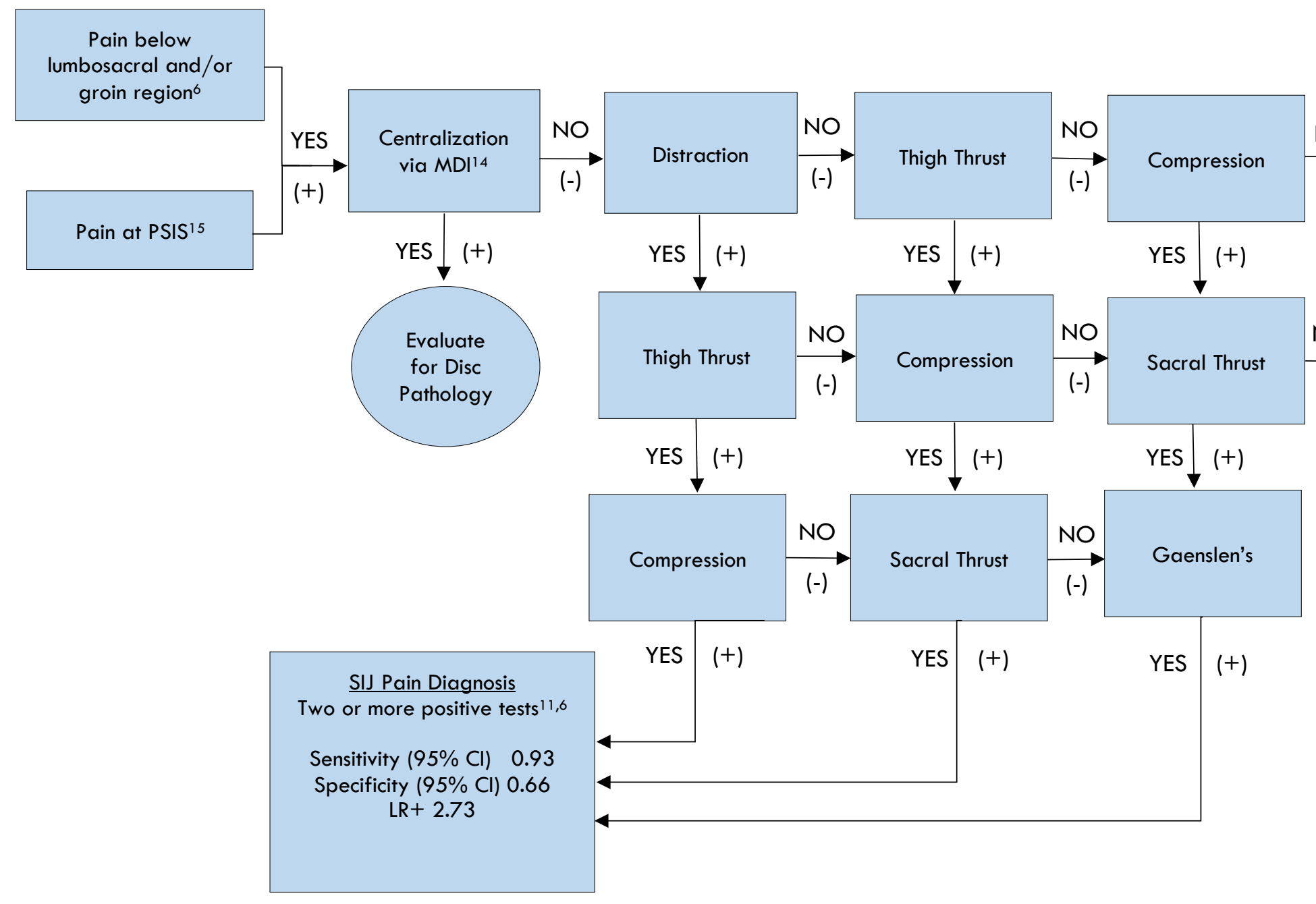

Evaluate

for Disc

Pathology

mbosacral and/o

oin region 6
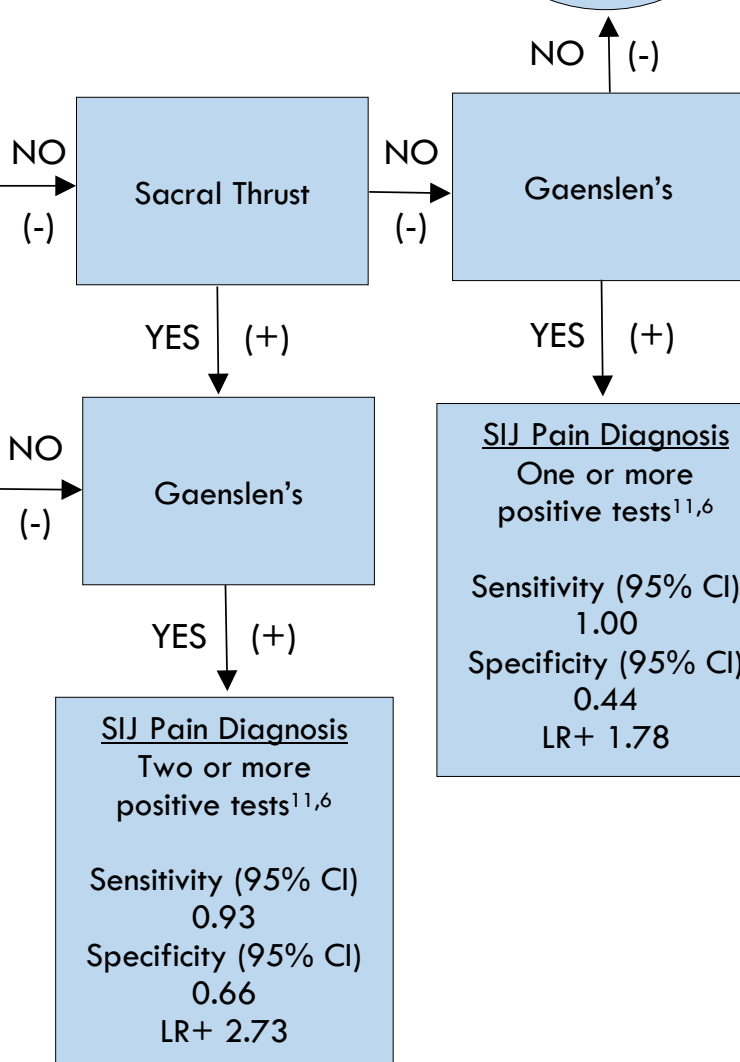
SIJ Pain Diagnosis One or more positive tests 11,6
Sensitivity $(95 \% \mathrm{Cl}$ 1.00
Specificity $(95 \% \mathrm{Cl})$
0.44
$\mathrm{LR}+1.78$

MDT: McKenzie Mechanical

Diagnosis and Therapy

$\mathrm{Cl}$ : Confidence Interval

LR+: Positive Likelihood

Ratio

23 